\title{
Integral experiments on thorium assemblies with D-T neutron source
}

\author{
Rong Liu ${ }^{a}$, Yiwei Yang, Song Feng, Lei Zheng, Caifeng Lai, Xinxin Lu, Mei Wang, and Li Jiang \\ Institute of Nuclear Physics and Chemistry, Key Laboratory of Neutron Physics, China Academy of Engineering Physics, Mianyang \\ 621900, Sichuan, China
}

\begin{abstract}
To validate nuclear data and code in the neutronics design of a hybrid reactor with thorium, integral experiments in two kinds of benchmark thorium assemblies with a D-T fusion neutron source have been performed. The one kind of 1D assemblies consists of polyethylene and depleted uranium shells. The other kind of 2D assemblies consists of three thorium oxide cylinders. The capture reaction rates, fission reaction rates, and $(\mathrm{n}, 2 \mathrm{n})$ reaction rates in ${ }^{232} \mathrm{Th}$ in the assemblies are measured by $\mathrm{ThO}_{2}$ foils. The leakage neutron spectra from the $\mathrm{ThO}_{2}$ cylinders are measured by a liquid scintillation detector. The experimental uncertainties in all the results are analyzed. The measured results are compared to the calculated ones with MCNP code and ENDF/B-VII.0 library data.
\end{abstract}

\section{Introduction}

The fusion-fission hybrid energy reactor (FFHER) for energy production, consisting of a low-power magnetic confinement fusion device and a blanket with subcritical fuel region, is one of advanced reactors of applying fusion technologies to solve the present energy crisis [1]. With the development of blanket technology, it is considered that thorium could be put in the blanket and used to breed ${ }^{233} \mathrm{U}$ by ${ }^{232} \mathrm{Th}$ capture reaction. Some conceptual blankets of FFHER based on the Th/U fuel cycle were designed [2]. The accuracy and reliability of evaluated nuclear data of thorium decide the validity of the physical design of the thorium-based blanket directly. Only a small number of integral neutronics experiments determining the ${ }^{232} \mathrm{Th}$ reaction rates are available in the literatures, and furthermore for some cases large differences between calculations and experiments can be observed [3]. In conceptual design stage, it is essential to perform the experiments for validating nuclear data and code [4].

The integral experiments on the thorium assemblies with a D-T neutron source have been performed in INPC [5-9]. In this paper, the progress in the integral experiments on representative assemblies is described. The ${ }^{232} \mathrm{Th}$ reaction rates in the assemblies and leakage neutron spectra are measured, separately. The measured results are compared to ones by using MCNP code with ENDF/BVII.0 library data.

\section{Thorium assemblies}

Aiming at researching on the nuclear data of thorium, the ways for establishing the assemblies are that the experiment is feasible for benchmark check and the material and neutron spectra are relevant to the conceptual

a e-mail: liurongzy@163.com design. The assemblies are classified as shells based on available materials and thorium oxide $\left(\mathrm{ThO}_{2}\right)$ cylinders.

\subsection{Polyethylene shell}

A polyethylene shell (PE), requiring only the use of standard cross sections $\mathrm{H}(\mathrm{n}, \mathrm{n})$ and $\mathrm{C}(\mathrm{n}, \mathrm{n})$ for interpretation of the results, is used for measuring the ${ }^{232} \mathrm{Th}$ reaction rates. The inner radius (IR) and the outer radius (OR) of the PE shell with density of $0.95 \mathrm{~g} / \mathrm{cm}^{3}$ are 80 and $230 \mathrm{~mm}$, respectively. The schematic diagram of the experimental assembly is shown in Fig. 1. Five slices of $\mathrm{ThO}_{2}$ (concentration $>99.95 \%$ ) foils are put in the radial channel at the $0^{\circ}$ direction to the incident $\mathrm{D}^{+}$beam. The mass and size of foils are about $4.2 \mathrm{~g}$ and $\varphi 30 \mathrm{~mm} \times 1 \mathrm{~mm}$.

A D-T fusion neutron source is located at the center of the assembly. The energy of $\mathrm{D}^{+}$ions is $225 \mathrm{keV}$ and $14-\mathrm{MeV}$ neutrons are produced by $\mathrm{D}^{+}$beam bombarding a TiT Target. The yield of the source is about $3 \times 10^{10}$ neutrons/s. The absolute yield is measured by the associated $\alpha$ particle method [10]. An Au-Si surface barrier semiconductor detector is at $178.2^{0}$ to $\mathrm{D}^{+}$beam in drift tube and used to measure the yield with LabVIEW-based auto-timing counter [11].

\subsection{Depleted uranium shell}

A depleted uranium (DU) shell in which fission nuclides are relevant to the fuel in the blanket [1] is adopted. The IR/OR of the DU $\left(\sim 99.6 \%{ }^{238} \mathrm{U}, \sim 0.4 \%{ }^{235} \mathrm{U}\right)$ shell is $131 \mathrm{~mm} / 300 \mathrm{~mm}$. The schematic diagram of the experimental assembly is shown in Fig. 2. Six slices of $\mathrm{ThO}_{2}$ samples are put in the radial channel at a $90^{\circ}$ direction to the incident $\mathrm{D}^{+}$beam. The $\mathrm{ThO}_{2}$ sample as a foil is that $\mathrm{ThO}_{2}$ powder is filled in a plexiglass box with inner diameter of $18 \mathrm{~mm}$ and outer diameter of $19 \mathrm{~mm}$. The mass of $\mathrm{ThO}_{2}$ powder is about $0.45 \mathrm{~g}$ and the thickness is about $0.7 \mathrm{~mm}$.

(C) The Authors, published by EDP Sciences. This is an Open Access article distributed under the terms of the Creative Commons Attribution License 4.0 (http://creativecommons.org/licenses/by/4.0/). 


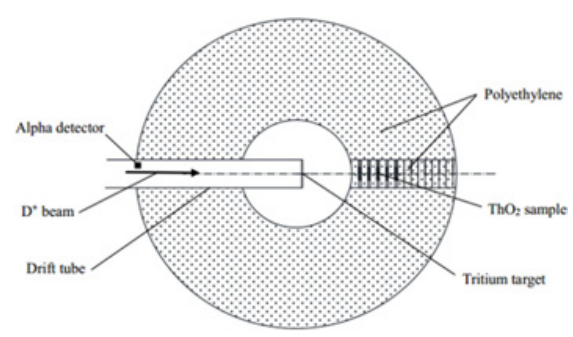

Figure 1. Polyethylene shell assembly.

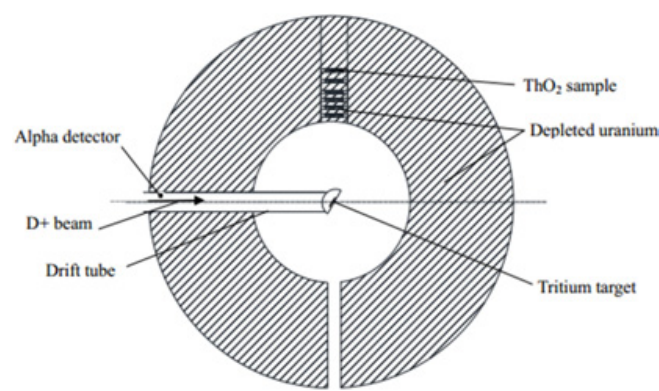

Figure 2. Depleted uranium shell assembly.

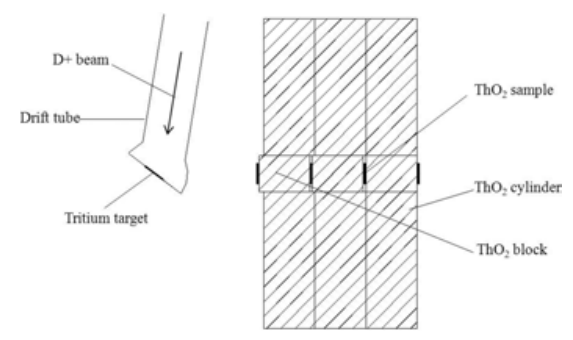

Figure 3. $\mathrm{ThO}_{2}$ cylindrical assembly.

\section{3. $\mathrm{ThO}_{2}$ cylinders}

The $\mathrm{ThO}_{2}$ benchmark assembly with the thickness of $150 \mathrm{~mm}$ is established and consists of three $\mathrm{ThO}_{2}$ cylinders with the thickness of $50 \mathrm{~mm}$ and the diameter of $300 \mathrm{~mm}$. The density and ingredient of these cylinders are described in Ref. [7]. The schematic diagram of $\mathrm{ThO}_{2}$ cylindrical assembly is shown in Fig. 3. The front surface of the assembly is $113 \mathrm{~mm}$ from the center of a target. Four slices of the $\mathrm{ThO}_{2}$ sample as described above are put in axial channel of the assembly.

\section{Results}

\section{1. ${ }^{232} \mathrm{Th}$ reaction rates in polyethylene shell}

The neutron induced reactions of ${ }^{232} \mathrm{Th}$ investigated are ${ }^{232} \mathrm{Th}(\mathrm{n}, \gamma),{ }^{232} \mathrm{Th}(\mathrm{n}, \mathrm{f})$ and ${ }^{232} \mathrm{Th}(\mathrm{n}, 2 \mathrm{n})$. The ${ }^{232} \mathrm{Th}$ reaction rates are obtained by using foil activation technique and an HPGe $\gamma$ spectrometer, and normalized to one source neutron and one ${ }^{232} \mathrm{Th}$ atom, as shown in Fig. 1.

The fertile nuclide ${ }^{232} \mathrm{Th}(\mathrm{n}, \gamma)^{233} \mathrm{Th}$ capture reaction rate (THCR) indicates breeding of fissile fuel ${ }^{233} \mathrm{U}$. THCR by measuring $311.98 \mathrm{keV} \gamma$ rays emitted from ${ }^{233} \mathrm{~Pa}$ (halflife $26.967 \mathrm{~d}$, from ${ }^{233} \mathrm{Th}$ decay), i.e., the production rate of ${ }^{233} \mathrm{U}\left({ }^{233} \mathrm{~Pa}\right.$ decay), can be deduced $[5,6]$.

The ${ }^{232} \mathrm{Th}(\mathrm{n}, \mathrm{f})$ (threshold $0.7 \mathrm{MeV}$ ) fission reaction rate (THFR) indicates energy amplification and neutron multiplication. THFR is obtained by using the fragment yield correction method. To estimate total fission rate, $151.16 \mathrm{keV} \gamma$ rays emitted from ${ }^{85 m} \mathrm{Kr}$ decay (half-life

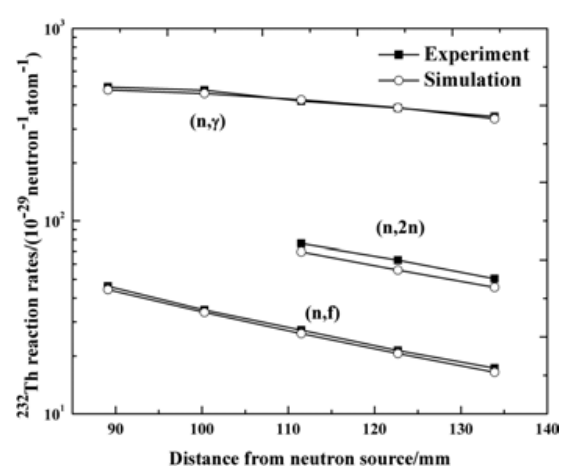

Figure 4. ${ }^{232}$ Th reaction rates in PE shell.

$4.48 \mathrm{hr}$ ) which is one of the fragments of ${ }^{232} \mathrm{Th}(\mathrm{n}, \mathrm{f})$ reaction are measured [7].

The ${ }^{232} \mathrm{Th}(\mathrm{n}, 2 \mathrm{n})^{231} \mathrm{Th}$ (threshold $6.5 \mathrm{MeV}$ ) reaction rate (THNR) indicates neutron multiplication. THNR is obtained by measuring $84.2 \mathrm{keV} \gamma$ rays emitted from ${ }^{231} \mathrm{Th}$ (half-life $25.52 \mathrm{hr}$ ) [8].

The ${ }^{232} \mathrm{Th}$ reaction rates are deduced from the measured activity by performing the appropriate corrections, which include fluctuations of the neutron flux during irradiation, detection efficiency, self-absorption of gamma ray in the foils, counting statistics and cited value of branching ratio. The experimental uncertainties are 3.0\% for THCR, $5.3 \%$ for THFR and $6.8 \%$ for THNR.

THCR, THFR and THNR are calculated by using MCNP code with ENDF/B-VII.0 library data. The model is completely consistent with the structure and ingredient of the assembly contained the target chamber and experimental hall. The angular dependences of the source neutron energy and intensity are calculated by "DROSG2000" code [12]. The $\mathrm{S}(\alpha, \beta)$ thermal scattering model in PE is considered. The calculated statistical uncertainty is less than $1 \%$. The ratio range and average values of calculation to experiment $(\mathrm{C} / \mathrm{E})$ are $0.96 \sim 1.02$ and 0.99 for THCR, $0.98 \sim 1.0$ and 0.99 for THFR, and $0.89 \sim$ 0.91 and 0.90 for THNR. The results state that both for THCR and THFR is well consistent within the range of experimental uncertainties, and calculations for THNR underestimate about $10 \%$. It is showed that the $\gamma$-ray offline method is feasible for determining the ${ }^{232} \mathrm{Th}$ reaction rates. The distributions of ${ }^{232} \mathrm{Th}$ reaction rates by the experiments and calculations are shown in Fig. 4.

The reaction rate ratio of ${ }^{232} \mathrm{Th}$ capture to fission gives fissile production rate in unit of fuel burn-up [9]. The relative ratios measured are about $10.76-20.17$ with the increase of radius in PE shell.

\section{2. ${ }^{232} \mathrm{Th}$ reaction rates in depleted uranium shell}

The ${ }^{232}$ Th reaction rates in DU shell are measured by the same method as described above and shown in Fig. 2. The experimental uncertainties are $3.1 \%$ for THCR, $5.3 \% \sim$ $5.5 \%$ for THFR $[8,9]$ and $6.8 \%$ for THNR. The ${ }^{232} \mathrm{Th}$ reaction rates are calculated by using MCNP code with ENDF/B-VII.0 library data. The range and average values of $\mathrm{C} / \mathrm{E}$ are $0.97 \sim 1.04$ and 1.02 for THCR, $0.95 \sim 1.02$ and 0.98 for THFR [7,9], and $1.07 \sim 1.12$ and 1.10 for THNR, respectively. The results state that the calculations and experiments are well consistent within the range of 


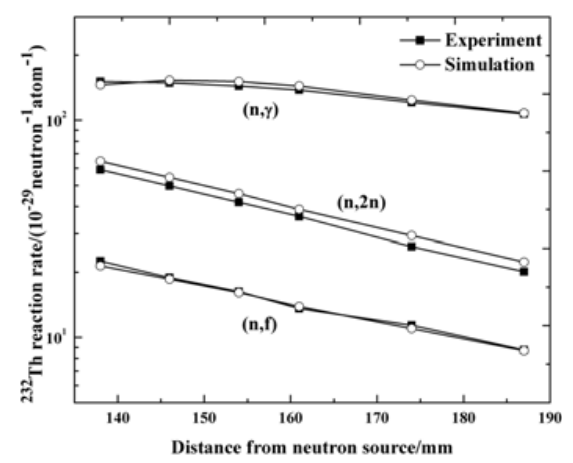

Figure 5. ${ }^{232} \mathrm{Th}$ reaction rates in depleted uranium shell.

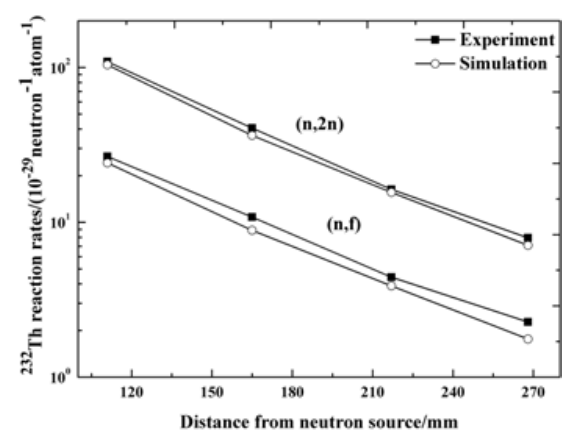

Figure 6. ${ }^{232} \mathrm{Th}$ reaction rates in $\mathrm{ThO}_{2}$ cylinder.

experimental uncertainties, except overestimating about $10 \%$ for THNR. The ratio of ${ }^{232}$ Th capture to fission are about $6.71 \sim 12.23$ with the increase of radius in DU shell. The ${ }^{232} \mathrm{Th}$ reaction rates by the experiments and calculations are shown in Fig. 5.

\subsection{Integral experiment in $\mathrm{ThO}_{2}$ cylinders}

\subsection{1. ${ }^{232}$ Th reaction rates}

The ${ }^{232} \mathrm{Th}(\mathrm{n}, \mathrm{f})$ and ${ }^{232} \mathrm{Th}(\mathrm{n}, 2 \mathrm{n})$ reaction rates in the $\mathrm{ThO}_{2}$ cylinder are measured by the same method as described above and shown in Fig. 3. The experimental uncertainties are $5.3 \% \sim 5.5 \%$ for THFR and $7.1 \%$ for THNR [8]. The ${ }^{232} \mathrm{Th}$ reaction rates are calculated by using MCNP code with ENDF/B-VII.0 library data. The range and average values of $\mathrm{C} / \mathrm{E}$ are $0.77 \sim 0.91$ and 0.85 for THFR, and $0.92 \sim 1.0$ and 0.96 for THNR [9], respectively. The results state that the calculations generally underestimate the experiments for THFR. The ${ }^{232} \mathrm{Th}$ reaction rates by the experiments and calculations are shown in Fig. 6.

\subsubsection{Leakage neutron spectra}

The neutron spectra leaking from the $\mathrm{ThO}_{2}$ cylinders are measured by the proton recoil method [4]. A $50.8 \mathrm{~mm}$ diameter and $50.8 \mathrm{~mm}$ length BC501A liquid scintillator coupled to a $50.8 \mathrm{~mm}$ diameter $9807 \mathrm{~B}$ photomultiplier of is used to measure recoil proton spectrum. The $n-\gamma$ pulse shape discrimination is based on the cross-zero method. The spectra are resolved by using iterative method, and their range is from $0.5 \mathrm{MeV}$ to $16 \mathrm{MeV}$. The detector is arranged in shielding room and at a $0^{0}$ direction to incident $\mathrm{D}^{+}$ion beam. The front surface of the assembly is $220 \mathrm{~mm}$ from the center of a target. The distance is $10.75 \mathrm{~m}$ from the detector to the neutron source. The

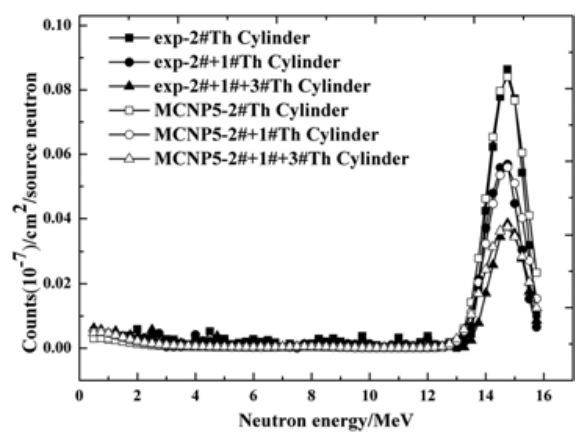

Figure 7. Leakage neutron spectra from $\mathrm{ThO}_{2}$ cylinders.

affect of background neutrons is negligible. The spectra from the three assemblies with thicknesses of 50,100 and $150 \mathrm{~mm}$ are measured, respectively, as shown in Fig. 3. The results are normalized to one source neutron and unit area. The experimental uncertainties are $9.7 \%$ for $0.5-1 \mathrm{MeV}, 6.7 \%$ for $1-3 \mathrm{MeV}$ and $6.3 \%$ for $3-16 \mathrm{MeV}$. The spectra are calculated by using MCNP code with ENDF/B-VII.0 library data. The results state that the experiments and calculations are generally consistent within the range of experimental uncertainties, and the spectra $(<5 \mathrm{MeV})$ should be analyzed further, as shown in Fig. 7.

\section{Conclusions}

To validate nuclear data and code in the neutronics design of blanket with thorium in FFHER, the integral experiments in the thorium assemblies with a D-T neutron source have been performed. The spherical assemblies based on available DU and $\mathrm{PE}$ shell, and the $\mathrm{ThO}_{2}$ cylinders are established. The distributions of ${ }^{232} \mathrm{Th}$ capture, fission and $(n, 2 n)$ reaction rates in the assemblies are measured, respectively. The leakage neutron spectra from $\mathrm{ThO}_{2}$ cylinders are measured. The experiments are compared to the calculations by using MCNP code with ENDF/B-VII.0 library data. The results state that the activation approach developed can work well for the experiments, and the ${ }^{232} \mathrm{Th}$ reaction rates are relevant to neutron spectra in assemblies. It is suggested that the integral experiments in a thorium assembly should be conducted further, and relevant cross section of ${ }^{232} \mathrm{Th}$ should be measured at CSNS white neutron source [13] in the future.

This work is supported by the Natural Science Foundation of China (No. 91226104, 11675155), the National Special Magnetic Confinement Fusion Energy Research, China (No.2015GB108001), and the National Key Research and Development Program of China (No. 2016YFA0401603).

\section{References}

[1] X. Shi, X. Peng, Nucl. Power Eng. 314, 5 (2010)

[2] J. Zhao, Y.W. Yang, Z.W. Zhou. et al., Fusion Eng. Des. 87, 1385 (2012)

[3] J. Adam, C. Bhatia, K. Katovsky, et al., Eur. Phys. J. A 47, 85 (2011) 
[4] R. Liu, T.H. Zhu, X.S. Yan, et al., Nucl. Data sheets 118, 588 (2014)

[5] Y.W. Yang, R. Liu, X.S. Yan, Acta Phys. Sin. 62, 032801 (2013)

[6] Y.W. Yang, R. Liu, L. Jiang, et al., Acta Phys. Sin. 63, 162801 (2014)

[7] S. Feng, R. Liu, X.X. Lu, et al., Acta Phys. Sin. 63, 162501 (2014)

[8] S. Feng, Y.W. Yang, X.X. Lu, R. Liu, et al., Ann. Nucl. Energy 81, 281 (2015)
[9] R. Liu , Y.W. Yang, X.S. Yan, et al., Ann. Nucl. Energy 92, 391 (2016)

[10] R. Liu, L.B. Lin, D.L. Wang, et al., Nucl. Elec. Det. Technol. 19, 428 (1999)

[11] J. Yan, R. Liu, C. Li, et al., Nucl. Sci. Technol. 20, 307 (2009)

[12] M. Drosg. IAEA-NDS-87, Rev. 8 (2003)

[13] H.T. Jing, J.Y. Tang, H.Q. Tang, et al., Nucl. Instrum. Methods Phys. A 621, 91 (2010) 